\title{
Robust Stability Analysis Based on LMI for Haptic Interface Systems with Uncertain Delay
}

\author{
Yanwen Liu $\mathbb{D},{ }^{1}$ Fanwei Meng $\mathbb{D},{ }^{2}$ Bowen Guan $\mathbb{D}{ }^{1}$, and Shuhao Zhang $\mathbb{D}^{1}$ \\ ${ }^{1}$ College of Automation, Harbin Engineering University, Harbin 150001, China \\ ${ }^{2}$ Department of Automation and Electrical Engineering, Northeastern University at Qinhuangdao, Qinhuangdao 066004, China
}

Correspondence should be addressed to Fanwei Meng; mengfanwei@neuq.edu.cn

Received 11 May 2018; Accepted 10 July 2018; Published 26 August 2018

Academic Editor: Liang $\mathrm{Hu}$

Copyright (C) 2018 Yanwen Liu et al. This is an open access article distributed under the Creative Commons Attribution License, which permits unrestricted use, distribution, and reproduction in any medium, provided the original work is properly cited.

\begin{abstract}
This paper presents the robust stability analysis based on LMI for force feedback haptic interface systems with uncertain delay. A full-order state observer is introduced to estimate states of the haptic device so as to directly obtain the feedback force, which expands the stability range of the system environment parameters when comparing with that of the system without an observer. By using the delay partitioning idea and the augmented state vector, the Lyapunov-Krasovskii functional is constructed, and then the new stability criterion based on LMI is given in the paper. With this new stability criterion, the observer gains can also be obtained directly. At last, a concrete example is used to prove the effectiveness and less conservativeness of this new approach.
\end{abstract}

\section{Introduction}

In recent years, the haptic interaction technique has been widely used in various fields such as remote surgery, virtual assembly, CAD (computer-aided design), maintenance in space, undersea or nuclear facilities, and telerobot and virtual prototyping [1]. The haptic interaction system is a typical force feedback system, which consists of three parts: a haptic device, a human operator, and a remote or virtual environment. The haptic device and the environment form a closed-loop feedback system, which is known as the haptic interface system $[2,3]$. For this system, stability is the basic requirement. Since people with subjective initiative are also included in the system and the instabilities may hurt people, the importance of stability for this kind of system becomes more important $[4,5]$.

Many researchers use passivity as an analysis and design tool for the haptic interface systems [1, 3, 6-8]. For example, Colgate et al. consider the passivity of a haptic system and derive the passivity condition by using the frequency domain lifting technique [3]. Colgate et al. give the same passivity condition by considering the whole haptic interface system as a pure discrete time system. Woo and Lee derive a passivity condition for haptic interface systems including the impedance of the operator [9]. By using the real frequency characteristics of the system, Liu et al. give new passivity and active boundaries [5]. Bianchini et al. present a passivity condition in terms of LMI [10].

Although these passive conditions are almost accepted by many researchers, the passive design is only a very conservative design approach, and the range of stability is generally much larger than that of the passivity $[11,12]$. Thus, for a stable human-computer interaction, the condition for passivity is only a sufficient but not necessary condition, and seeking for a less conservative stability condition becomes a crucial issue to researchers [13-16].

It needs to be emphasized that for most of the control systems, especially for computer control systems, there are usually various time delays due to program calculation, data processing, and communication [15]. Similarly, there are also such delays in the haptic interface systems, which as a major source of instability may not be ignored. Therefore, to study the stability of the system, we must consider the influence of time delays [17]. In order to obtain a more conservative delay-dependent stability criterion for the system with time delays, many methods have been proposed based on the Lyapunov-Krasovskii functional [18-21]. By far, researchers have made significant progress in this field. For example, 
the descriptor system approach [22], the free-weighting matrix technique $[23,24]$, and the delay partitioning approach [25-27].

In this paper, the haptic interface system with a virtual environment for virtual wall implementation is studied. This system is in fact a typical sampled-data control system with an uncertain time-varying delay; thus, most of the research on such system is based on the discrete time control theory. For instance, based on LMIs, Dang et al. give the stability conditions of the haptic interface systems implemented by the virtual wall $[2,13,15,17]$. In literatures $[13,15]$, observers are also used to directly obtain the states of the haptic device, but the state estimate errors are not included in the Lyapunov-Krasovskii functional and the observer gains cannot be directly obtained by solving the stability criterion in terms of LMIs (linear matrix inequality). Allowing this, a full-order state observer is used in this paper to estimate the states of the haptic device so as to calculate the force feedback $[1,3,12]$. The observer gains can be obtained by solving the stability criteria in terms of LMI. An augmented vector composed of states and state estimate errors is defined and used in the Lyapunov-Krasovskii functional candidate. Moreover, the utilization of the delay partitioning idea may further improve the conservatism of the stability condition given in this paper.

Other parts of the paper are arranged as follows. Section 2 gives the structure diagram and the model of the haptic interface system. In Section 3, the discretized state space model for the plant is constructed, and the full-order state observer is presented. Section 4 gives the mathematical preliminaries used in the paper. By using the delay partitioning idea and the augmented state vector, a novel Lyapunov-Krasovskii functional is constructed in Section 5, and then the new stability criterion based on LMI is given in this section. In Section 6, the effectiveness and less conservativeness of the proposed approach are testified through a simulation example. The conclusions are given in Section 7.

\section{Model of the System}

Figure 1 shows the structure diagram of the haptic system, which is a one degree of freedom system and with an environment for stiff virtual wall implementation. In Figure 1, the haptic device is equivalent to the continuous plant, and the virtual wall environment is equivalent to the discrete controller. $t_{d}$ denotes the uncertain delay. For the convenience of the following analysis, we use the discrete-time form delay by defining $d=t_{d} / T$ and assuming that $d(k)$ satisfies $1 \leq d_{m} \leq$ $d(k) \leq d_{M}$, where $d_{m}$ and $d_{M}$ are both constant positive scalars. $H$ is a $\mathrm{ZOH}$ (zero order hold), $f$ denotes the force signal exerted by a human operator, $q$ is the displacement output of the haptic device, $u$ is the feedback signal from the environment, which is also a force signal, and $T$ is the sampling period.

Since a human operator is also a part of the haptic interface system, its dynamic model is also considered in some studies. Simulation and experimental results in the literature $[7,11,12,14,16]$ show that the human operator may actually make the system more stable, and this conclusion can be

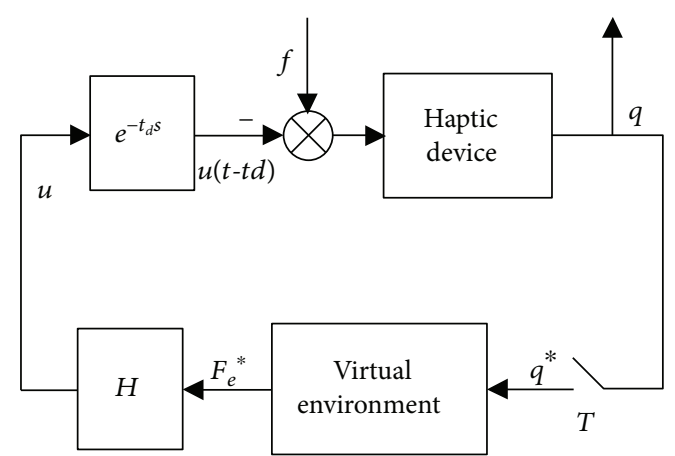

FIgURE 1: Structure diagram of the haptic interface system.

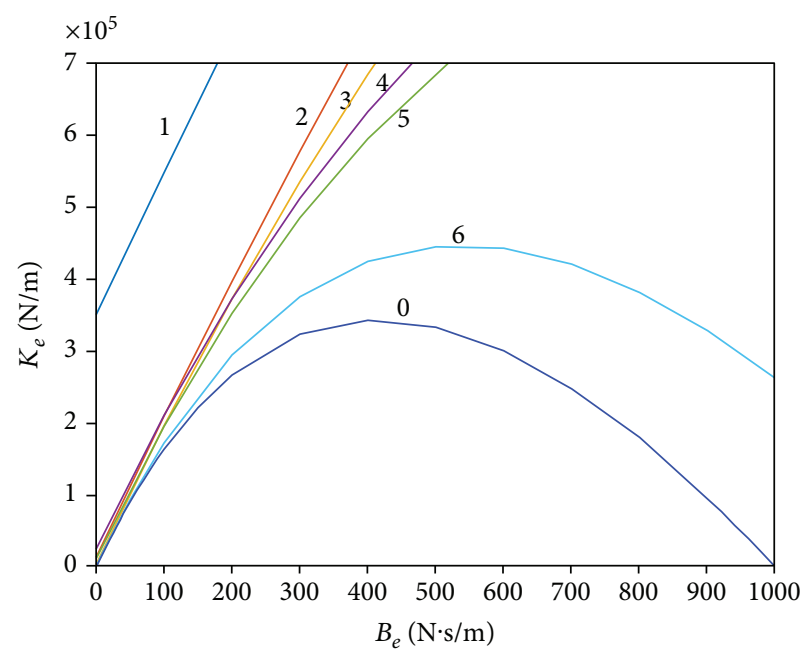

FIgURE 2: Stability regions in the $B-K$ parametric plane (without delay).

further proved by Figure 2 in this paper. Therefore, the dynamic model of the human operator can be ignored in the stability analysis of the haptic interface system.

If the human operator model is considered and it is assumed that the mass of the haptic device is $m$, the damping coefficient of the haptic device is $b$, the mass of the human operator is $m_{\mathrm{h}}$, the damping coefficient of the human operator is $b_{\mathrm{h}}$, the stiffness coefficient of the human operator is $k_{\mathrm{h}}$, then the dynamic model of the human operator and haptic device can be described as

$$
\left(m+m_{\mathrm{h}}\right) \ddot{q}+\left(b+b_{\mathrm{h}}\right) \dot{q}+k_{\mathrm{h}} q=u+f .
$$

In view of the above analysis, the human operator model will be ignored in the following parts of the paper. Thus, the dynamic model of the haptic device (plant) is described as $x_{1}=q$

$$
m \ddot{q}+b \dot{q}=u+f .
$$

The state vector $\mathbf{x}=\left[\mathbf{x}_{1} \mathbf{x}_{2}\right]^{T}$ is chosen as and $x_{2}=\dot{q}=v$ ( $v$ is the velocity of the haptic device); the output is chosen as $y=x_{1}=q$; then the state space expression for the haptic device is 


$$
\begin{aligned}
& \dot{\mathbf{x}}=A \mathbf{x}+B_{1} u+B_{2} f, \\
& \mathbf{y}=C \mathbf{x},
\end{aligned}
$$

where

$$
\begin{aligned}
& A=\left[\begin{array}{cc}
0 & 1 \\
0 & -\frac{b}{m}
\end{array}\right], \\
& B_{1}=B_{2}=\left[\begin{array}{l}
0 \\
1 \\
m
\end{array}\right], \\
& C=\left[\begin{array}{ll}
1 & 0
\end{array}\right] .
\end{aligned}
$$

For the stiff virtual wall environment, it is composed of a virtual spring and a damper in mechanical parallel. Defining the stiffness of the spring as $K_{e}$ and the damping coefficient of the damper as $B_{e}$ and assuming the position of the virtual wall is equal to zero, the virtual force is then given by

$$
F_{e}= \begin{cases}K_{e} q+B_{e} \dot{q}, & \text { contact } \\ 0, & \text { noncontact }\end{cases}
$$

where $q$ in (5) is the displacement of the haptic device.

\section{Realization of the State Observer}

In order to obtain the discrete state observer, we should first derive the discretized model of the haptic device. By combining $\mathrm{ZOH}$, the sampler, and the delay $d(k)$ with the haptic device, we can get the following discretized model of the haptic device:

$$
\begin{aligned}
\mathbf{x}(k+1) & =A_{d} \mathbf{x}(k)+B_{1 d} u(k-d(k))+B_{2 d} f(k), \\
\mathbf{y}(k) & =C_{d} \mathbf{x}(k),
\end{aligned}
$$

where

$$
\begin{aligned}
& A_{d}=e^{A T}, \\
& B_{1 d}=B_{2 d}=\int_{0}^{T} e^{A t} B_{1} d t \\
& C_{d}=C .
\end{aligned}
$$

Since the discrete-time signals $q^{*}$ and $v^{*}$ are needed to compute the feedback force $F_{e}^{*}$, a full-order state observer is used here to estimate the discrete-time states $\left(q^{*}\right.$ and $\left.v^{*}\right)$ of the haptic device.

According to the system model in expression (6), the fullorder state observer is of the following form:

$$
\begin{aligned}
\widehat{\mathbf{x}}(k+1) & =A_{d} \widehat{\mathbf{x}}(k)+B_{1 d} u(k-d(k))+L_{d}[\mathbf{y}(k)-\widehat{\mathbf{y}}(k)], \\
\widehat{\mathbf{y}}(k) & =C_{d} \widehat{\mathbf{x}}(k) .
\end{aligned}
$$

Defining the state estimation error as $\mathbf{e}(k)=\mathbf{x}(k)-\widehat{\mathbf{x}}(k)$, we can get the following dynamic equation:

$$
\mathbf{e}(k+1)=\left(A_{d}-L_{d} C_{d}\right) \mathbf{e}(k)+B_{2 d} f(k) .
$$

Considering the following control law:

$$
u(k)=-K_{d} \widehat{\mathbf{x}}(k)=-\left[\begin{array}{ll}
K_{e} & B_{e}
\end{array}\right] \widehat{\mathbf{x}}(k) .
$$

And defining the augmented vector as

$$
\boldsymbol{a}(k)=\left[\begin{array}{l}
\mathbf{x}(k) \\
\mathbf{e}(k)
\end{array}\right] .
$$

The following augmented closed-loop system can be obtained:

$$
\begin{aligned}
\boldsymbol{a}(k+1) & =\tilde{A} \boldsymbol{\alpha}(k)+\tilde{B}_{1} u(k-d(k))+\tilde{B}_{2} f(k), \\
\mathbf{y}(k) & =\tilde{C} \boldsymbol{\alpha}(k),
\end{aligned}
$$

where

$$
\begin{aligned}
\tilde{A} & =\left[\begin{array}{cc}
A_{d} & \mathbf{0} \\
\mathbf{0} & A_{d}-L_{d} C_{d}
\end{array}\right], \\
\tilde{B}_{1} & =\left[\begin{array}{cc}
-B_{1 d} K_{d} & B_{1 d} K_{d} \\
\mathbf{0} & \mathbf{0}
\end{array}\right], \\
\tilde{B}_{2} & =\left[\begin{array}{c}
B_{2 d} \\
B_{2 d}
\end{array}\right], \\
\tilde{C} & =\left[\begin{array}{ll}
C_{d} & \mathbf{0}
\end{array}\right] .
\end{aligned}
$$

From expression (12), we can get the discrete-time state space (14), which will be used for the next asymptotic stability analysis:

$$
\begin{aligned}
{\left[\begin{array}{c}
x(k+1) \\
e(k+1)
\end{array}\right]=} & {\left[\begin{array}{cc}
A_{d} & \mathbf{0} \\
\mathbf{0} & A_{d}-L_{d} C_{d}
\end{array}\right]\left[\begin{array}{l}
x(k) \\
e(k)
\end{array}\right] } \\
& +\left[\begin{array}{cc}
-B_{1 d} K_{d} & B_{1 d} K_{d} \\
\mathbf{0} & \mathbf{0}
\end{array}\right]\left[\begin{array}{c}
x(k-d(k)) \\
e(k-d(k))
\end{array}\right] .
\end{aligned}
$$

\section{Mathematical Preliminaries}

The following lemmas are prepared for the next derivations.

Lemma 1 [28]. For the given matrices $X$ and $Y$ with appropriate dimensions, one has

$$
X^{T} Y+Y^{T} X \leq \varepsilon X^{T} X+\varepsilon^{-1} Y^{T} Y, \quad \forall \varepsilon>0 .
$$

Lemma 2 [13]. For any symmetric positive definite matrix $R \in \mathbb{R}^{n \times n}$, integers $q \geq p>0$, and vector function $x:[p, q] \rightarrow$ $\mathbb{R}^{n}$, the following inequality holds

$$
-(q-p+1) \sum_{i=p}^{q} x^{T}(i) R x(i) \leq\left[\sum_{i=p}^{q} x(i)\right]^{T}(-R)\left[\sum_{i=p}^{q} x(i)\right] .
$$


Lemma 3 (Schur complement) [29]. Given the constant matrices $M, N$, and $Q$ with appropriate dimensions, where $M^{T}=M$ and $Q^{T}=Q>0$, then $M+N^{T} Q^{-1} N<0$ if and only if

$$
\left[\begin{array}{cc}
M & N^{T} \\
N & -Q
\end{array}\right]<0,
$$

or equivalently

$$
\left[\begin{array}{ll}
-Q & N \\
N^{T} & M
\end{array}\right]<0 .
$$

$$
\left[\begin{array}{ccccccccc}
\Omega_{11}^{\prime} & 0 & 0 & 0 & Z_{1} & 0 & 0 & 0 & 0 \\
* & \Omega_{22}^{\prime} & 0 & 0 & 0 & 0 & 0 & 0 & 0 \\
* & * & -R_{3}-2 Z_{2} & 0 & 0 & 0 & 0 & 0 & Z_{2} \\
* & * & * & -R_{4} & 0 & 0 & 0 & 0 & 0 \\
* & * & * & * & Q_{3}-Q_{1}-Z_{1} & 0 & 0 & 0 & 0 \\
* & * & * & * & * & Q_{4}-Q_{2} & 0 & 0 & 0 \\
* & * & * & * & * & * & Q_{5}-Q_{3} & 0 & 0 \\
* & * & * & * & * & * & * & Q_{6}-Q_{4} & 0 \\
* & * & * & * & * & * & * & * & -Q_{5}-Z_{2} \\
* & * & * & * & * & * & * & * & * \\
* & * & * & * & * & * & * & * & * \\
* & * & * & * & * & * & * & * & * \\
* & * & * & * & * & * & * & * & * \\
* & * & * & * & * & * & * & * & * \\
* & * & * & * & * & * & * & * & * \\
* & * & * & * & * & * & * & * & * \\
* & * & * & * & * & * & * & * & * \\
* & * & * & * & * & * & * & * & *
\end{array}\right.
$$

where $\rho=d_{M}-d_{m}=d_{M}-3 \tau$.

$$
\begin{aligned}
& \Omega_{11}^{\prime}=-P_{1}+Q_{1}+R_{1}+(\rho+1) R_{3}-Z_{1}, \\
& \Omega_{22}^{\prime}=\varepsilon^{-1} A_{d}^{T} A_{d}-P_{2}+Q_{2}+R_{2}+(\rho+1) R_{4} .
\end{aligned}
$$

Proof. Firstly, by using the delay partitioning idea [25], the lower bound of delay $d(k)$ can be described as $d_{m}=N \tau$, where $N$ and $\tau$ are integers. Then one can express the time delay $d(k)$ as $d(k)=d_{m}+\delta(k)=N \tau+\delta(k)$, where $\delta(k) \in\left[0, d_{M}-N \tau\right]$. In the following derivations, one chooses $d_{m}=3 \tau(N=3)$ to divide $d_{m}$ into three parts.

Now, one chose the Lyapunov-Krasovskii functional as

$$
V(k)=\sum_{i=1}^{7} V_{i}(k),
$$

where

\section{Main Results}

A novel Lyapunov-Krasovskii functional is constructed for the discrete-time haptic interface system (14), and then a new stability criterion based on LMI will be given in this section. The main result is as follows.

Theorem 1. For the given positive integers $\tau$ and $d_{M}$, the discrete-time system (14) is asymptotically stable if the LMI in inequality (19) holds. Where the variables should satisfy $\varepsilon>0$ and $P_{1}, P_{2}, Q_{1}, Q_{2}, Q_{3}, Q_{4}, Q_{5}, Q_{6}, R_{1}, R_{2}, R_{3}, R_{4}, Z_{1}$, and $Z_{2}$ are positive matrices with appropriate dimensions,

$$
\left.\begin{array}{cccccccc}
0 & 0 & A_{d}^{T} P_{1} & \left(A_{d}-I\right)^{T} Z_{1} & \left(A_{d}-I\right)^{T} Z_{2} & 0 & 0 & 0 \\
0 & 0 & 0 & 0 & 0 & A_{d}^{T} P & C_{d}^{T} V & C_{d}^{T} V \\
Z_{2} & 0 & -K_{d}^{T} B_{1 d}^{T} P_{1} & -K_{d}^{T} B_{1 d}^{T} Z_{1} & -K_{d}^{T} B_{1 d}^{T} Z_{2} & 0 & 0 & 0 \\
0 & 0 & K_{d}^{T} B_{1 d}^{T} P_{1} & K_{d}^{T} B_{1 d}^{T} Z_{1} & K_{d}^{T} B_{1 d}^{T} Z_{2} & 0 & 0 & 0 \\
0 & 0 & 0 & 0 & 0 & 0 & 0 & 0 \\
0 & 0 & 0 & 0 & 0 & 0 & 0 & 0 \\
0 & 0 & 0 & 0 & 0 & 0 & 0 & 0 \\
0 & 0 & 0 & 0 & 0 & 0 & 0 & 0 \\
0 & 0 & 0 & 0 & 0 & 0 & 0 & 0 \\
0 & 0 & 0 & 0 & 0 & 0 & 0 & 0 \\
-R_{1}-Z_{2} & 0 & 0 & 0 & 0 & 0 & 0 & 0 \\
* & -R_{2} & 0 & 0 & 0 & 0 & 0 & 0 \\
* & * & -P_{1} & 0 & 0 & 0 & 0 & 0 \\
* & * & * & -\frac{1}{\tau^{2}} Z_{1} & 0 & 0 & 0 & 0 \\
* & * & * & * & -\frac{1}{\rho^{2}} Z_{2} & 0 & 0 & 0 \\
* & * & * & * & * & -P_{2} & 0 & 0 \\
* & * & * & * & * & * & -P_{2} & 0 \\
* & * & * & * & * & -\varepsilon^{-1} I
\end{array}\right]
$$

$$
\begin{aligned}
& V_{1}(k)=\alpha^{T}(k)\left[\begin{array}{cc}
P_{1} & 0 \\
0 & P_{2}
\end{array}\right] \alpha(k), \\
& V_{2}(k)=\sum_{i=k-\tau}^{k-1}\left[\begin{array}{lll}
\alpha^{T}(i) & \alpha^{T}(i-\tau) & \alpha^{T}(i-2 \tau)
\end{array}\right] \\
& {\left[\begin{array}{cccccc}
Q_{1} & 0 & 0 & 0 & 0 & 0 \\
0 & Q_{2} & 0 & 0 & 0 & 0 \\
0 & 0 & Q_{3} & 0 & 0 & 0 \\
0 & 0 & 0 & Q_{4} & 0 & 0 \\
0 & 0 & 0 & 0 & Q_{5} & 0 \\
0 & 0 & 0 & 0 & 0 & Q_{6}
\end{array}\right]\left[\begin{array}{c}
\alpha(i) \\
\alpha(i-\tau) \\
\alpha(i-2 \tau)
\end{array}\right],} \\
& V_{3}(k)=\sum_{i=k-d_{M}}^{k-1} \alpha^{T}(i)\left[\begin{array}{cc}
R_{1} & 0 \\
0 & R_{2}
\end{array}\right] \alpha(i),
\end{aligned}
$$




$$
\begin{aligned}
& V_{4}(k)=\sum_{i=k-d(k)}^{k-1} \alpha^{T}(i)\left[\begin{array}{cc}
R_{3} & 0 \\
0 & R_{4}
\end{array}\right] \alpha(i), \\
& V_{5}(k)=\sum_{j=d_{M}+1}^{-d_{m}} \sum_{i=k+j}^{k-1} \alpha^{T}(i)\left[\begin{array}{cc}
R_{3} & 0 \\
0 & R_{4}
\end{array}\right] \alpha(i), \\
& V_{6}(k)=\tau \sum_{j=-\tau}^{-1} \sum_{i=k+j}^{k-1} \Delta x^{T}(i) Z_{1} \Delta x(i), \\
& V_{7}(k)=\left(d_{M}-d_{m}\right) \sum_{j=-d_{M}}^{-d_{m}-1} \sum_{i=k+j}^{k-1} \Delta x^{T}(i) Z_{2} \Delta x(i), \\
& \Delta x(k)=x(k+1)-x(k) .
\end{aligned}
$$

Defining $\Delta V(k)=V(k+1)-V(k)$. Then by using the state-space (14), one can get

$$
\begin{aligned}
\Delta V_{1}(k)= & x^{T}(k+1) P_{1} x(k+1)-x^{T}(k) P_{1} x(k) \\
& +e^{T}(k+1) P_{2} e(k+1)-e^{T}(k) P_{2} e(k) \\
= & x^{T}(k)\left[A_{d}^{T} P_{1} A_{d}-P_{1}\right] x(k)-2 x^{T}(k) A_{d}^{T} P_{1} B_{1 d} K_{d} x \\
& \cdot(k-d(k))+2 x^{T}(k) A_{d}^{T} P_{1} B_{1 d} K_{d} e(k-d(k)) \\
& +x^{T}(k-d(k)) K_{d}^{T} B_{1 d}^{T} P_{1} B_{1 d} K_{d} x(k-d(k)) \\
& -2 x^{T}(k-d(k)) K_{d}^{T} B_{1 d}^{T} P_{1} B_{1 d} K_{d} e(k-d(k)) \\
& +e^{T}(k-d(k)) K_{d}^{T} B_{1 d}^{T} P_{1} B_{1 d} K_{d} e(k-d(k)) \\
& +e(k)\left[A_{d}^{T} P_{2} A_{d}-P_{2}-A_{d}^{T} P_{2} L_{d} C_{d}\right. \\
& \left.\quad-C_{d}^{T} L_{d}^{T} P_{2} A_{d}+C_{d}^{T} L_{d}^{T} P_{2} L_{d} C_{d}\right] e(k),
\end{aligned}
$$

By using Lemma 1 , one has

$$
\begin{aligned}
& \Delta V_{1}(k) \leq x^{T}(k)\left[A_{d}^{T} P_{1} A_{d}-P_{1}\right] x(k)-2 x^{T}(k) A_{d}^{T} P_{1} B_{1 d} K_{d} x \\
& \cdot(k-d(k))+2 x^{T}(k) A_{d}^{T} P_{1} B_{1 d} K_{d} e(k-d(k)) \\
&+x^{T}(k-d(k)) K_{d}^{T} B_{1 d}^{T} P_{1} B_{1 d} K_{d} x(k-d(k)) \\
&- 2 x^{T}(k-d(k)) K_{d}^{T} B_{1 d}^{T} P_{1} B_{1 d} K_{d} e(k-d(k)) \\
&+ e^{T}(k-d(k)) K_{d}^{T} B_{1 d}^{T} P_{1} B_{1 d} K_{d} e(k-d(k)) \\
&+e(k)\left[A_{d}^{T} P_{2} A_{d}-P_{2}+\varepsilon C_{d}^{T} L_{d}^{T} P_{2} P_{2} L_{d} C_{d}\right. \\
&\left.+\varepsilon^{-1} A_{d}^{T} A_{d}+C_{d}^{T} L_{d}^{T} P_{2} L_{d} C_{d}\right] e(k),
\end{aligned}
$$

$$
\begin{aligned}
\Delta V_{2}(k)= & x^{T}(k) Q_{1} x(k)+e^{T}(k) Q_{2} e(k)+x^{T}(k-\tau) \\
& \cdot\left(Q_{3}-Q_{1}\right) x(k-\tau)+e^{T}(k-\tau)\left(Q_{4}-Q_{2}\right) e(k-\tau) \\
& +x^{T}(k-2 \tau)\left(Q_{5}-Q_{3}\right) x(k-2 \tau)+e^{T}(k-2 \tau) \\
& \cdot\left(Q_{6}-Q_{4}\right) e(k-2 \tau)-x^{T}\left(k-d_{m}\right) Q_{5} x\left(k-d_{m}\right) \\
& -e^{T}\left(k-d_{m}\right) Q_{6} e\left(k-d_{m}\right),
\end{aligned}
$$

$$
\begin{aligned}
\Delta V_{3}(k)= & x^{T}(k) R_{1} x(k)+e^{T}(k) R_{2} e(k)-x^{T}\left(k-d_{M}\right) R_{1} x \\
& \cdot\left(k-d_{M}\right)-e^{T}\left(k-d_{M}\right) R_{2} e\left(k-d_{M}\right),
\end{aligned}
$$

$$
\begin{aligned}
\Delta V_{4}(k)= & \sum_{i=k+1-d(k+1)}^{k+1} \alpha^{T}(i)\left[\begin{array}{cc}
R_{3} & 0 \\
0 & R_{4}
\end{array}\right] \alpha(i) \\
& -\sum_{i=k-d(k)}^{k} \alpha^{T}(i)\left[\begin{array}{cc}
R_{3} & 0 \\
0 & R_{4}
\end{array}\right] \alpha(i) \\
\leq & \alpha^{T}(k)\left[\begin{array}{cc}
R_{3} & 0 \\
0 & R_{4}
\end{array}\right] \alpha(k)-\alpha^{T}(k-d(k))
\end{aligned}
$$

$$
\begin{aligned}
& {\left[\begin{array}{cc}
R_{3} & 0 \\
0 & R_{4}
\end{array}\right] \alpha(k-d(k)) } \\
& +\sum_{i=k-d_{M}+1}^{k-d_{m}} \alpha^{T}(i)\left[\begin{array}{cc}
R_{3} & 0 \\
0 & R_{4}
\end{array}\right] \alpha(i), \\
\Delta V_{5}(k)= & \left(d_{M}-d_{m}\right) \alpha^{T}(k)\left[\begin{array}{cc}
R_{3} & 0 \\
0 & R_{4}
\end{array}\right] \alpha(k) \\
& -\sum_{i=k-d_{M}+1}^{k-d_{m}} \alpha^{T}(i)\left[\begin{array}{ll}
R_{3} & 0 \\
0 & R_{4}
\end{array}\right] \alpha(i) .
\end{aligned}
$$

Define $\rho=d_{M}-d_{m}$, then from (27) and (28), one can get

$$
\begin{aligned}
\Delta V_{4}(k) & +\Delta V_{5}(k) \\
\leq & x^{T}(k)\left[(\rho+1) R_{3}\right] x(k) \\
& +e^{T}(k)\left[(\rho+1) R_{4}\right] e(k) \\
& -x^{T}(k-d(k)) R_{3} x(k-d(k)) \\
& -e^{T}(k-d(k)) R_{4} e(k-d(k)) .
\end{aligned}
$$

By using Lemma 2, one has

$$
\begin{aligned}
\Delta V_{6}(k)= & \tau^{2} \Delta x^{T}(k) Z_{1} \Delta x(k)-\tau \sum_{i=k-\tau}^{k-1} \Delta x^{T}(i) Z_{1} \Delta x(i) \\
\leq & \tau^{2} x^{T}(k)\left(A_{d}-I\right)^{T} Z_{1}\left(A_{d}-I\right) x(k) \\
& -2 \tau^{2} x^{T}(k)\left(A_{d}-I\right)^{T} Z_{1} B_{1 d} K_{d} x(k-d(k)) \\
& +2 \tau^{2} x^{T}(k)\left(A_{d}-I\right)^{T} Z_{1} B_{1 d} K_{d} e(k-d(k)) \\
& +\tau^{2} x^{T}(k-d(k)) K_{d}^{T} B_{1 d}^{T} Z_{1} B_{1 d} K_{d} x(k-d(k)) \\
& -2 \tau^{2} x^{T}(k-d(k)) K_{d}^{T} B_{1 d}^{T} Z_{1} B_{1 d} K_{d} e(k-d(k)) \\
& +\tau^{2} e^{T}(k-d(k)) K_{d}^{T} B_{1 d}^{T} Z_{1} B_{1 d} K_{d} e(k-d(k)) \\
& -\left[\begin{array}{c}
x(k) \\
x(k-\tau)
\end{array}\right]^{T}\left[\begin{array}{cc}
-Z_{1} & Z_{1} \\
Z_{1} & -Z_{1}
\end{array}\right]\left[\begin{array}{c}
x(k) \\
x(k-\tau)
\end{array}\right] .
\end{aligned}
$$

Similarly, by using Lemma 2 , one can get 


$$
\begin{aligned}
& \Delta V_{7}(k)=\rho^{2} \Delta x^{T}(k) Z_{2} \Delta x(k)-\rho \sum_{j=k-d(k)}^{k-d_{m}-1} \Delta x^{T}(i) Z_{2} \Delta x(i) \\
& -\rho \sum_{j=k-d_{M}}^{k-d(k)-1} \Delta x^{T}(i) Z_{2} \Delta x(i) \\
& \xi^{T}(k)=\left[x^{T}(k), e^{T}(k), x^{T}(k-d(k)),\right. \\
& e^{T}(k-d(k)), x^{T}(k-\tau), e^{T}(k-\tau), \\
& x^{T}(k-2 \tau), e^{T}(k-2 \tau), x^{T}\left(k-d_{m}\right), \\
& \left.e^{T}\left(k-d_{m}\right), x^{T}\left(k-d_{M}\right), e^{T}\left(k-d_{M}\right)\right] \text {. }
\end{aligned}
$$$$
\leq \rho^{2} x^{T}(k)\left(A_{d}-I\right)^{T} Z_{2}\left(A_{d}-I\right) x(k)
$$$$
-2 \rho^{2} x^{T}(k)\left(A_{d}-I\right)^{T} Z_{2} B_{1 d} K_{d} x(k-d(k))
$$$$
+2 \rho^{2} x^{T}(k)\left(A_{d}-I\right)^{T} Z_{2} B_{1 d} K_{d} e(k-d(k))
$$$$
+\rho^{2} x^{T}(k-d(k)) K_{d}^{T} B_{1 d}^{T} Z_{2} B_{1 d} K_{d} x(k-d(k))
$$$$
-2 \rho^{2} x^{T}(k-d(k)) K_{d}^{T} B_{1 d}^{T} Z_{2} B_{1 d} K_{d} e(k-d(k))
$$$$
+\rho^{2} e^{T}(k-d(k)) K_{d}^{T} B_{1 d}^{T} Z_{2} B_{1 d} K_{d} e(k-d(k))
$$$$
-\left[\begin{array}{c}
x\left(k-d_{M}\right) \\
x(k-d(k))
\end{array}\right]^{T}\left[\begin{array}{cc}
-Z_{2} & Z_{2} \\
Z_{2} & -Z_{2}
\end{array}\right]\left[\begin{array}{c}
x\left(k-d_{M}\right) \\
x(k-d(k))
\end{array}\right]
$$$$
-\left[\begin{array}{c}
x(k-d(k)) \\
x\left(k-d_{m}\right)
\end{array}\right]^{T}\left[\begin{array}{cc}
-Z_{2} & Z_{2} \\
Z_{2} & -Z_{2}
\end{array}\right]\left[\begin{array}{c}
x(k-d(k)) \\
x\left(k-d_{m}\right)
\end{array}\right] \text {. }
$$

Then, from (23), (24), (25), (26), (27), (28), (29), (30), and (31), one has

$$
\Delta V(k)=\sum_{i=1}^{7} \Delta V_{i}(k) \leq \xi^{T}(k) \Omega \xi(k)
$$

where

Denoting

$$
\Omega=\left[\begin{array}{cccccccccccc}
\Omega_{11} & 0 & \Omega_{13} & \Omega_{14} & Z_{1} & 0 & 0 & 0 & 0 & 0 & 0 & 0 \\
* & \Omega_{22} & 0 & 0 & 0 & 0 & 0 & 0 & 0 & 0 & 0 & 0 \\
* & * & \Omega_{33} & \Omega_{34} & 0 & 0 & 0 & 0 & Z_{2} & 0 & Z_{2} & 0 \\
* & * & * & \Omega_{44} & 0 & 0 & 0 & 0 & 0 & 0 & 0 & 0 \\
* & * & * & * & Q_{3}-Q_{1}-Z_{1} & 0 & 0 & 0 & 0 & 0 & 0 & 0 \\
* & * & * & * & * & Q_{4}-Q_{2} & 0 & 0 & 0 & 0 & 0 & 0 \\
* & * & * & * & * & * & Q_{5}-Q_{3} & 0 & 0 & 0 & 0 & 0 \\
* & * & * & * & * & * & * & Q_{6}-Q_{4} & 0 & 0 & 0 & 0 \\
* & * & * & * & * & * & * & * & -Q_{5}-Z_{2} & 0 & 0 & 0 \\
* & * & * & * & * & * & * & * & * & -Q_{6} & 0 & 0 \\
* & * & * & * & * & * & * & * & * & * & -R_{1}-Z_{2} & 0 \\
* & * & * & * & * & * & * & * & * & * & * & -R_{2}
\end{array}\right],
$$

with

$$
\begin{aligned}
\Omega_{11}= & A_{d}^{T} P_{1} A_{d}-P_{1}+Q_{1}+R_{1}+(\rho+1) R_{3} \\
& +\tau^{2}\left(A_{d}-I\right)^{T} Z_{1}\left(A_{d}-I\right)-Z_{1} \\
& +\rho^{2}\left(A_{d}-I\right)^{T} Z_{2}\left(A_{d}-I\right), \\
\Omega_{13}= & -A_{d}^{T} P_{1} B_{1 d} K_{d}-\left(A_{d}-I\right)^{T} Z_{1} B_{1 d} K_{d} \\
& -\left(A_{d}-I\right)^{T} Z_{2} B_{1 d} K_{d},
\end{aligned}
$$

$$
\begin{aligned}
\Omega_{14}= & A_{d}^{T} P_{1} B_{1 d} K_{d}+\left(A_{d}-I\right)^{T} Z_{1} B_{1 d} K_{d} \\
& +\left(A_{d}-I\right)^{T} Z_{2} B_{1 d} K_{d}, \\
\Omega_{22}= & A_{d}^{T} P_{2} A_{d}+\varepsilon C_{d}^{T} L_{d}^{T} P_{2} P_{2} L_{d} C_{d}+\varepsilon^{-1} A_{d}^{T} A_{d} \\
& +C_{d}^{T} L_{d}^{T} P_{2} L_{d} C_{d}-P_{2}+Q_{2}+R_{2}+(\rho+1) R_{4}, \\
\Omega_{33}= & K_{d}^{T} B_{1 d}^{T} P_{1} B_{1 d} K_{d}-R_{3}+K_{d}^{T} B_{1 d}^{T} Z_{1} B_{1 d} K_{d} \\
& +K_{d}^{T} B_{1 d}^{T} Z_{2} B_{1 d} K_{d}-2 Z_{2},
\end{aligned}
$$




$$
\begin{aligned}
\Omega_{34}= & -K_{d}^{T} B_{1 d}^{T} P_{1} B_{1 d} K_{d}-K_{d}^{T} B_{1 d}^{T} Z_{1} B_{1 d} K_{d} \\
& -K_{d}^{T} B_{1 d}^{T} Z_{2} B_{1 d} K_{d}, \\
\Omega_{44}= & K_{d}^{T} B_{1 d}^{T} P_{1} B_{1 d} K_{d}+K_{d}^{T} B_{1 d}^{T} Z_{1} B_{1 d} K_{d} \\
& +K_{d}^{T} B_{1 d}^{T} Z_{2} B_{1 d} K_{d}-R_{4} .
\end{aligned}
$$

Then, the delay-dependent stability condition for system (14) is $\Omega<0$.

Defining $L_{d}^{T}=V P_{2}^{-1}$, then the condition $\Omega<0$ is equivalent to the inequality (19) by using the Schur complement (Lemma 3). This completes the proof.

Remark 1. In the Lyapunov-Krasovskii functional $V(k)$, the summation term $V_{4}(k)$ and double summation term $V_{5}(k)$ use the same matrix variables $R_{3}$ and $R_{4}$. On one hand, the number of matrices that need to be solved is reduced, and the total amount of calculation is reduced. On the other hand, in the process of solving the forward difference $\Delta V_{4}(k)$ and $\Delta V_{5}(k)$, the terms

$$
+\sum_{i=k-d_{M}+1}^{k-d_{m}} \alpha^{T}(i)\left[\begin{array}{cc}
R_{3} & 0 \\
0 & R_{4}
\end{array}\right] \alpha(i)
$$

and

$$
-\sum_{i=k-d_{M}+1}^{k-d_{m}} \alpha^{T}(i)\left[\begin{array}{cc}
R_{3} & 0 \\
0 & R_{4}
\end{array}\right] \alpha(i),
$$

cancelled each other, which simplifies the final result of Theorem 1.

Remark 2. By introducing the partitioned delay $\tau$ into the double summation term $V_{6}(k)$, the time delay information of the system is fully utilized, and it is expected that the results obtained in this paper are less conservative.

Remark 3. The double summation term $V_{7}(k)$ introduces the time delay bound information $d_{m}$ and $d_{M}$. During the process of solving the forward difference $\Delta V_{7}(k)$, the term $-\rho \sum_{j=k-d M}^{k-d_{m}-1} \Delta x^{T}(i) Z_{2} \Delta x(i)$ is divided into two parts: $-\rho \sum_{j=k-d(k)}^{k-d_{m}-1} \Delta x^{T}(i) Z_{2} \Delta x(i)$ and $-\rho \sum_{j=k-d_{M}}^{k-d(k)-1} \Delta x^{T}(i) Z_{2} \Delta x(i)$, then the time delay information $d(k)$ is increased, which is expected to reduce the possible conservatism. This can be further proved by the following examples.

\section{Numerical Simulation Results}

We adopt the parameters of the haptic interface in literatures $[3,5,30]: m=0.5 \mathrm{~kg}$ and $b=0.1 \mathrm{~N} \cdot \mathrm{s} / \mathrm{m}$. Supposing the sampling period is $T=0.001 \mathrm{~s}$.

According to expression (3), the continuous plant of the system is given by

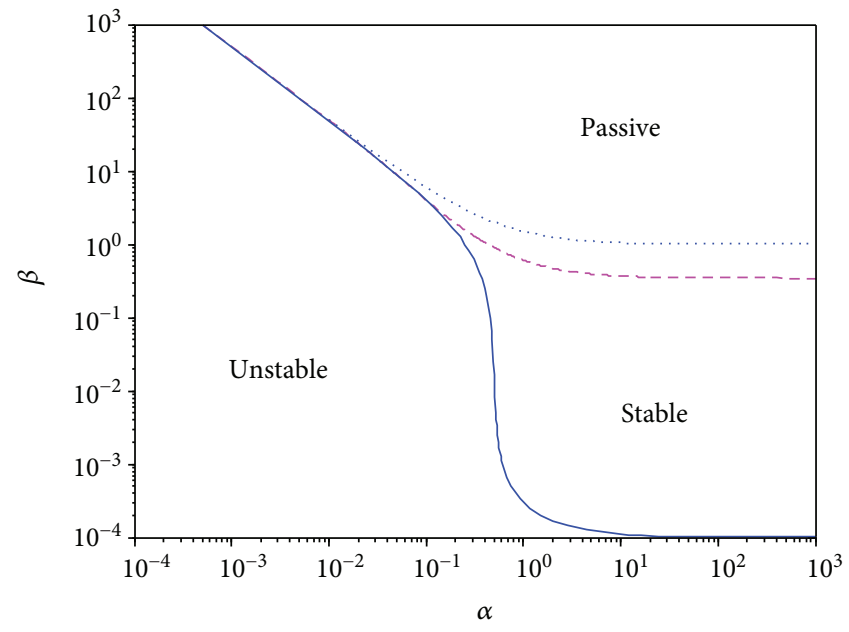

FIgURe 3: Passivity and stability regions in the $\alpha-\beta$ plane (without delay).

$$
\begin{aligned}
& \dot{\mathbf{x}}=\left[\begin{array}{cc}
0 & 1 \\
0 & -0.2
\end{array}\right] \mathbf{x}+\left[\begin{array}{l}
0 \\
2
\end{array}\right] u+\left[\begin{array}{l}
0 \\
2
\end{array}\right] f, \\
& \mathbf{y}=\left[\begin{array}{ll}
1 & 0
\end{array}\right] \mathbf{x} .
\end{aligned}
$$

With

$$
\begin{aligned}
& A=\left[\begin{array}{cc}
0 & 1 \\
0 & -0.2
\end{array}\right], \\
& B=\left[\begin{array}{l}
0 \\
2
\end{array}\right], \\
& C=\left[\begin{array}{ll}
1 & 0
\end{array}\right] .
\end{aligned}
$$

According to (6), the corresponding discretized state space matrixes are

$$
\begin{aligned}
A_{d} & =\left[\begin{array}{cc}
1 & 0.9999 \times 10^{-3} \\
0 & 0.9998
\end{array}\right], \\
B_{d} & =\left[\begin{array}{l}
0.9999 \times 10^{-6} \\
0.1999 \times 10^{-2}
\end{array}\right], \\
C_{d} & =\left[\begin{array}{ll}
1 & 0
\end{array}\right] .
\end{aligned}
$$

The special case of $d(k)=0$ is firstly considered here. For comparison with the passivity conditions in previous literatures, we define two dimensionless parameters $\alpha=B_{e} /\left(K_{e} T\right)$ and $\beta=b / B_{e}$. The passivity and stability regions in the $\alpha-\beta$ plane with $d(k)=0$ are shown in Figure 3. Where the dotted line is the passivity bound provided by the literature $[3,30]$, the dashed line is the passivity bound by the literature [5], and the solid line shows the stability bound. From Figure 3, we can conclude that the passivity domain is only a small region contained in the stability domain.

To further testify the influence of the human operator model, the stability regions of the system with different operator models are given in Figure 2. Parameters $[11,14]$ for the 
TABLE 1: Parameters for different operator models $[11,14]$.

\begin{tabular}{lccc}
\hline Model & $m_{\mathrm{h}}(\mathrm{kg})$ & $b_{\mathrm{h}}(\mathrm{N} \cdot \mathrm{s} / \mathrm{m})$ & $k_{\mathrm{h}}(\mathrm{N} / \mathrm{m})$ \\
\hline 1 & 17.51 & 175.12 & 175.12 \\
2 & 4.54 & 6.83 & 12.5 \\
3 & 2.0 & 2.0 & 10.0 \\
4 & 1.0 & 12.6 & 39.5 \\
5 & 0.8 & 5.5 & 568 \\
6 & 0.15 & 0.5 & 7.0 \\
\hline
\end{tabular}

TABle 2: Allowable maximum $d_{M}$ for different $\tau$.

\begin{tabular}{llcc}
\hline$d_{m}$ & 3 & 6 & 9 \\
$\tau$ & 1 & 2 & 3 \\
$d_{M}$ & 6 & 10 & 15 \\
\hline
\end{tabular}

human operator model are shown in Table 1 . The curve 0 in Figure 2 is the stability region for the system without the human operator model for $d(k)=0$, and curves 1 to 6 denotes the stability regions for different operator parameters in Table 1. As can be seen from Figure 2, the stability region for the system without considering the human operator model is the smallest one. That is to say, the human operator can actually make the system more stable.

We now add the time-varying delay $d(k)$ into the haptic system. Parameters of the feedback control law (virtual wall) in expression (10) are selected as $K_{e}=760, B_{e}=40(\alpha \approx 52$ and $\beta=0.0025$ ). Supposing that the lower delay bound is $d_{m}=3 \tau$. According to the main results of Theorem 1 in this paper, we can see that system (14) is asymptotically stable for $d_{M} \leq 15$. The allowable maximum $d_{M}$ for different $\tau$ is presented in Table 2.

The observer gains for different cases are as follows:

$$
\begin{aligned}
& L_{d 1}=\left[\begin{array}{ll}
2.651 & 1748
\end{array}\right]^{T}, \quad d_{m}=3, \tau=1, d_{M}=6, \\
& L_{d 2}=\left[\begin{array}{ll}
2.996 & 2220
\end{array}\right]^{T}, \quad d_{m}=6, \tau=2, d_{M}=10, \\
& L_{d 3}=\left[\begin{array}{ll}
3.7117 & 3442
\end{array}\right]^{T}, \quad d_{m}=9, \tau=3, d_{M}=15 .
\end{aligned}
$$

Introducing the maximum delay $d_{M}=15$, the real and observed states (displacement $q$ and velocity $v$ ) of the haptic interface system under the sinusoidal input of $f(t)$ are shown in Figures 4 and 5. In Figure 5, curve 0 denotes the real velocity and curves 1 to 3 denote the observed velocity with the observer gain $L_{d 1}$ to $L_{d 3}$, respectively. From Figures 4 and 5, we can see that the minimum state estimate errors are obtained by using the observer gains $L_{d 3}$ in (42). Thus, the observer gains $L_{\mathrm{d} 3}$ will be used in the following simulations.

Figure 6 shows the stability regions in the $B-K$ parametric plane with different $d_{M}$. As can be seen from Figure 6 , the maximum time delay is closely related to the stability of the system. The larger the upper delay bound $d_{M}$ is, the smaller is the stability region.

Table 3 lists the maximum stiffness $K_{e}$ and the corresponding damping coefficient $B_{e}$ under different $d_{M}$. As can be seen from Figure 3, Table 3, and Figure 6, the allowable virtual wall parameters are far greater than the passive ones.

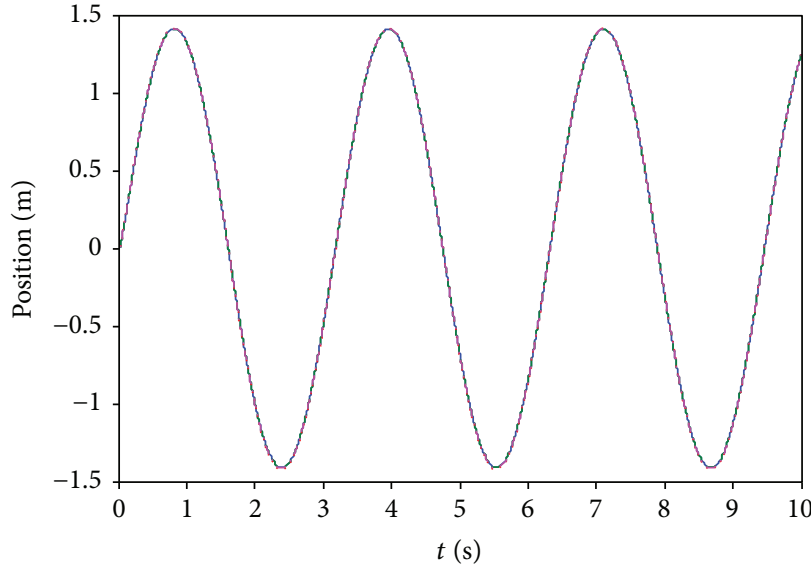

FIGURE 4: Real and observed position $q$ of the haptic device.

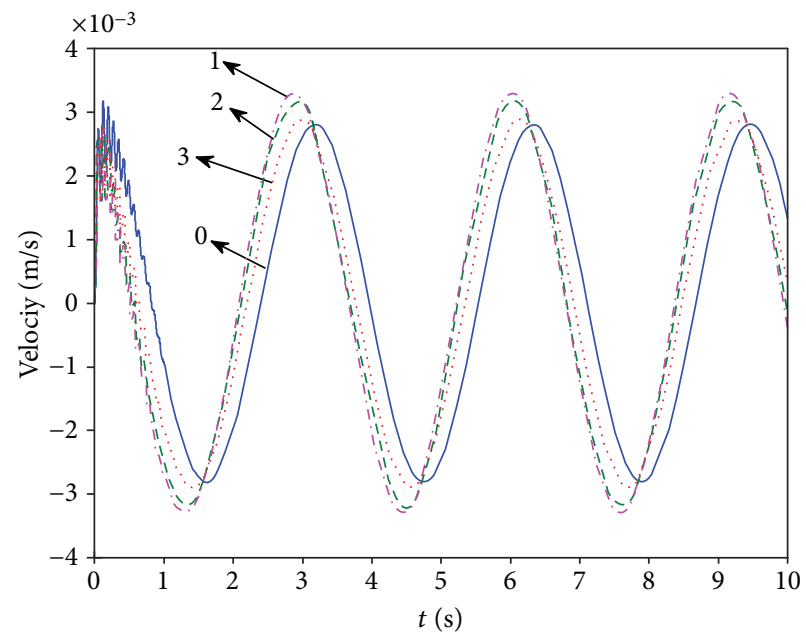

FIgURE 5: Real and observed velocity $v$ of the haptic device.

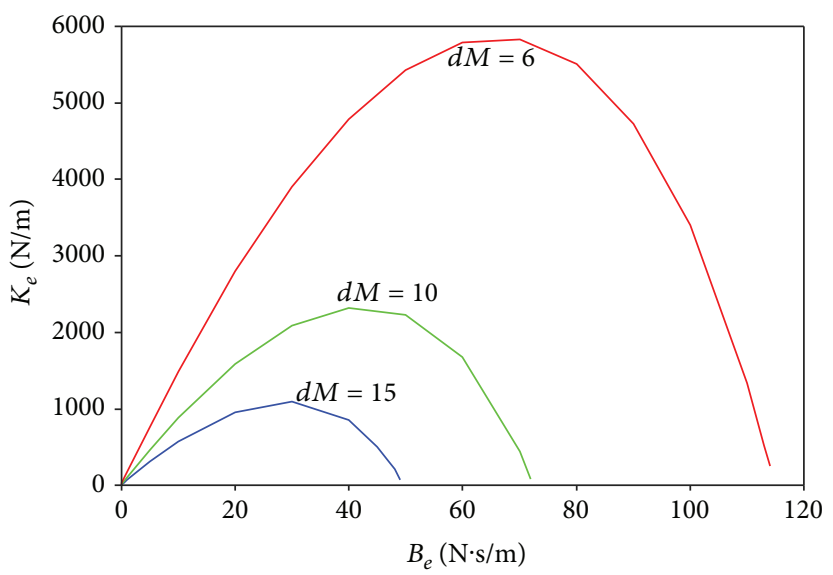

FIgURE 6: Stability regions in the $B-K$ parametric plane.

To further testify advantages of the stability criterion proposed in this paper, we also use other methods in existing literatures for the above plant (40). Table 4 lists the allowable maximum delay upper bounds $d_{M}$ for different 
TABle 3: Maximum $K_{e}$ for different $d_{M}$.

\begin{tabular}{lccc}
\hline$d_{M}$ & 6 & 10 & 15 \\
\hline$K_{e}(\mathrm{~N} / \mathrm{m})$ & 5830 & 2320 & 1097 \\
$B_{e}(\mathrm{~N} \cdot \mathrm{s} / \mathrm{m})$ & 70 & 40 & 30 \\
\hline
\end{tabular}

TABle 4: Maximum $d_{M}$ for different $d_{m}$.

\begin{tabular}{lcccc}
\hline$d_{m}$ & 3 & 6 & 9 & 12 \\
\hline Ibrir et al. [28] & 5 & 8 & 11 & 13 \\
Zhang et al. [19] & 6 & 8 & 12 & 13 \\
Dang et al. [15] & 6 & 9 & 13 & 14 \\
Theorem 1 & 6 & 10 & 15 & 15 \\
\hline
\end{tabular}

$d_{m}$. From Table 4 , we can see that the results by using the approach proposed in this paper are obviously superior to those of the existing literatures, so the advantages and less conservativeness of the stability criterion given in this paper are further proved.

\section{Conclusions}

This paper presents the robust stability analysis based on LMI for force feedback haptic interface systems with uncertain delay. By using the delay partitioning approach and augmented vector composed of states and state estimate errors, a novel less conservative stability criterion is obtained based on LMI. Through a concrete haptic example, it can be concluded that the passive condition is only a small region contained in the stability domain, and the maximum time delay has a great effect on the stability of the system. By comparing with the results available in the existing literatures, the advantages and less conservativeness of the stability criterion given in this paper are further proved.

\section{Data Availability}

The data used to support the findings of this study are available from the corresponding author upon request.

\section{Conflicts of Interest}

The authors declare no conflict of interest.

\section{Acknowledgments}

This work is supported by the National Natural Science Foundation of China (no. 61304076), the Youth Foundation of Hebei Educational committee (no. ZD2016203) and the Doctoral Foundation of Liaoning Province (no. 20170520333).

\section{References}

[1] A. Haddadi and K. H. Zad, "Stability analysis of haptic interfaces for different types of sampled signals and virtual environment implementations," in 2010 IEEE Haptics Symposium, pp. 293-299, Waltham, MA, USA, March 2010.
[2] Q. V. Dang, A. Dequidt, L. Vermeiren, and M. Dambrine, "Experimental study on stability of a haptic system with variable time delays," 2014 IEEE/ASME International Conference on Advanced Intelligent Mechatronics, 2014, pp. 554-559, Besacon, France, July 2014.

[3] J. E. Colgate and G. Schenkel, "Passivity of a class of sampleddata systems: application to haptic interfaces," Journal of Robotic Systems, vol. 14, no. 1, pp. 37-47, 1997.

[4] J. E. Colgate, P. E. Grafing, M. C. Stanley, and G. Schenkel, "Implementation of stiff virtual walls in force-reflecting interfaces," in Proceedings of IEEE Virtual Reality Annual International Symposium, pp. 202-208, Seattle, WA, USA, September 1993.

[5] Y. W. Liu, J. Li, Z. He, and G. X. Wang, "Passivity design of a haptic interface for the remote servo manipulator," Control Theory \& Applications, vol. 28, no. 7, pp. 994-998, 2011.

[6] J. Hoogen, R. Riener, and G. Schmidt, "Control aspects of a robotic haptic interface for kinesthetic knee joint simulation," Control Engineering Practice, vol. 10, no. 11, pp. 1301-1308, 2002.

[7] R. J. Adams and B. Hannaford, "Stable haptic interaction with virtual environments," IEEE Transactions on Robotics and Automation, vol. 15, no. 3, pp. 465-474, 1999.

[8] J. E. Colgate and G. Schenkel, "Passivity of a class of sampleddata systems: application to haptic interfaces," in American Control Conference, 1994, pp. 3236-3240, Baltimore, MD, USA, June-July 1994.

[9] H. S. Woo and D. Y. Lee, "Passivity analysis of a 1-DOF haptic system with consideration of human arm impedance," in 2008 International Conference on Control, Automation and Systems, pp. 1133-1137, Seoul, South Korea, October 2008.

[10] G. Bianchini, M. Orlandesi, and D. Prattichizzo, "An LMI framework for analysis and design of multi-dimensional haptic systems," in 2008 47th IEEE Conference on Decision and Control, pp. 4564-4569, Cancun, Mexico, December 2008.

[11] J. J. Gil, A. Avello, Á. Rubio, and J. Flórez, "Stability analysis of a 1 DOF haptic interface using the Routh-Hurwitz criterion," IEEE Transactions on Control Systems Technology, vol. 12, no. 4, pp. 583-588, 2004.

[12] T. Hulin, A. A. Schaffer, and G. Hirzinger, "Passivity and stability boundaries for haptic systems with time delay," IEEE Transactions on Control Systems Technology, vol. 22, no. 4, pp. 1297-1309, 2014.

[13] Q. V. Dang, L. Vermeiren, A. Dequidt, and M. Dambrine, "Augmented observer approach for high-impedance haptic system with time delay," Mathematics and Computers in Simulation, vol. 113, pp. 51-68, 2015.

[14] Q. V. Dang, A. Dequidt, L. Vermeiren, A. Fratu, and M. Dambrine, "Stability analysis of haptic interfaces: effects of dynamic parameters," in Proceedings of the Remote Engineering and Virtual instrumentation Conference-REV, pp. 137-143, Brasov, Romania, June-July 2011.

[15] Q. V. Dang, L. Vermeiren, A. Dequidt, and M. Dambrine, "Experimental study on the stability of an impedance-type force-feedback architecture based on an augmented-state observer for a haptic system under time delay using a LMI approach," Proceedings of the Institution of Mechanical Engineers, Part I: Journal of Systems and Control Engineering, vol. 230, no. 1, pp. 58-71, 2016.

[16] J. J. Gil, M. J. Puerto, I. Diaz, and E. Sanchez, "On the z-width limitation due to the vibration modes of haptic interfaces," in 
2010 IEEE/RSJ International Conference on Intelligent Robots and Systems, pp. 5054-5059, Taipei, Taiwan, October 2010.

[17] Q. V. Dang, L. Vermeiren, A. Dequidt, and M. Dambrine, "Improved LMI conditions for stability analysis of discretetime haptic system under time-varying communication delays," in 2012 International Conference on Control, Automation and Information Sciences (ICCAIS), pp. 271-276, Ho Chi Minh City, Vietnam, November 2012.

[18] W. Zhang, J. Wang, Y. Liang, and Z. Han, "Improved delay-range-dependant stability criterion for discrete-time systems with interval time varying delay," Journal of Information \& Computational Science, vol. 14, no. 8, pp. 33213328, 2011.

[19] N. Zhang, Y. Fan, and X. He, "Observer based Ho output tracking control for discrete time systems with multiple time varying delays," Journal of Zhejiang University of Technology, vol. 43, no. 4, pp. 438-444, 2015.

[20] C. Singkibud and K. Mukdasai, "Improved delay-rangedependant stability criteria for discrete-time linear systems with interval time-varying delay and nonlinear perturbations," International Journal of Pure and Applied Mathematics, vol. 93, no. 3, pp. 427-447, 2014.

[21] K. Ramakrishnan and G. Ray, "Robust stability criteria for a class of uncertain discrete-time systems with time-varying delay," Applied Mathematical Modelling, vol. 37, no. 3, pp. 1468-1479, 2013.

[22] E. Fridman and U. Shaked, "Stability and guaranteed cost control of uncertain discrete delay systems," International Journal of Control, vol. 78, no. 4, pp. 235-246, 2005.

[23] B. Zhang, S. Xu, and Y. Zou, "Improved stability criterion and its applications in delayed controller design for discretetime systems," Automatica, vol. 44, no. 11, pp. 2963-2967, 2008.

[24] H. Shao and Q. L. Han, "New stability criteria for linear discrete-time systems with interval-like time-varying delays," IEEE Transactions on Automatic Control, vol. 56, no. 3, pp. 619-625, 2011.

[25] X. Meng, J. Lam, B. Du, and H. Gao, "A delay-partioning approach to the stability analysis of discrete-time systems," Automatica, vol. 46, no. 3, pp. 610-614, 2010.

[26] Q. L. Han, "A discrete delay decomposition approach to stability of linear retarded and neutral systems," Automatica, vol. 45, no. 2, pp. 517-524, 2009.

[27] J. J. Hun, X. Y. Kong, H. X. Zhang, and X. Zhou, "Delay-partitioning approach for systems with interval time-varying delay and nonlinear perturbations," Journal of Computational and Applied Mathematics, vol. 281, pp. 74-81, 2015.

[28] S. Ibrir and S. Diopt, "Novel LMI conditions for observerbased stabilization of lipschitzian nonlinear systems and uncertain linear systems in discrete-time," Applied Mathematics and Computation, vol. 206, no. 2, pp. 579-588, 2008.

[29] M. Luo, S. Zhong, R. Wang, and W. Kang, "Robust stability analysis for discrete-time stochastic neural networks systems with time-varying delays," Applied Mathematics and Computation, vol. 209, no. 2, pp. 305-313, 2009.

[30] M. Fardad and B. Bamieh, "A necessary and sufficient frequency domain criterion for the passivity of SISO sampleddata systems," IEEE Transactions on Automatic Control, vol. 54, no. 3, pp. 611-614, 2009. 


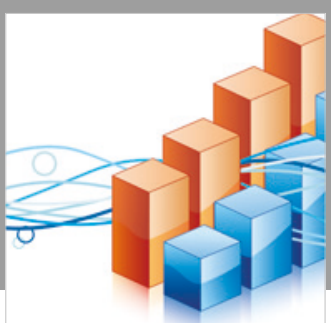

Advances in

Operations Research

\section{-n-m}
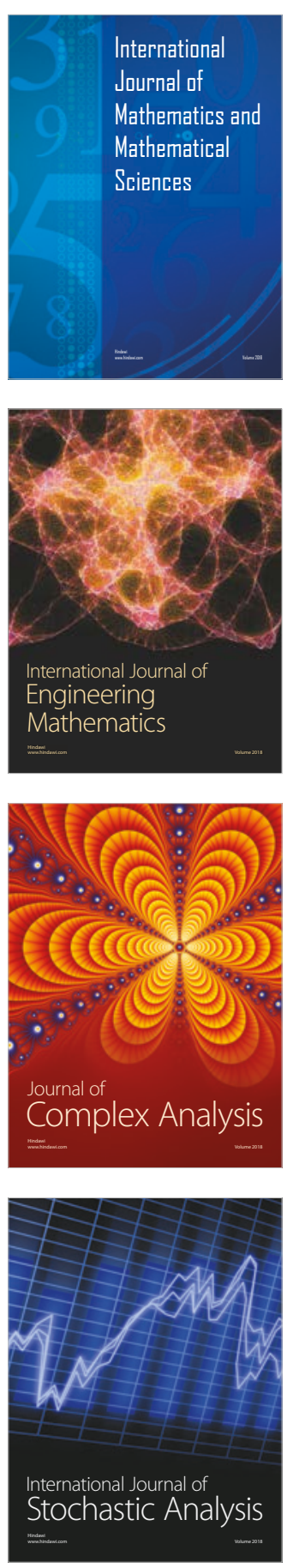
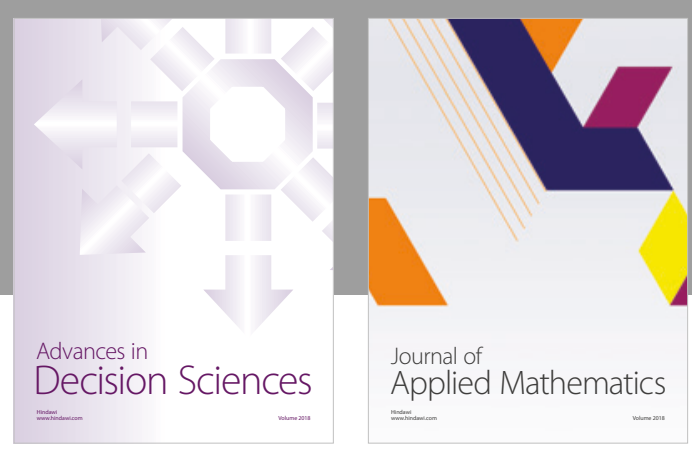

Journal of

Applied Mathematics
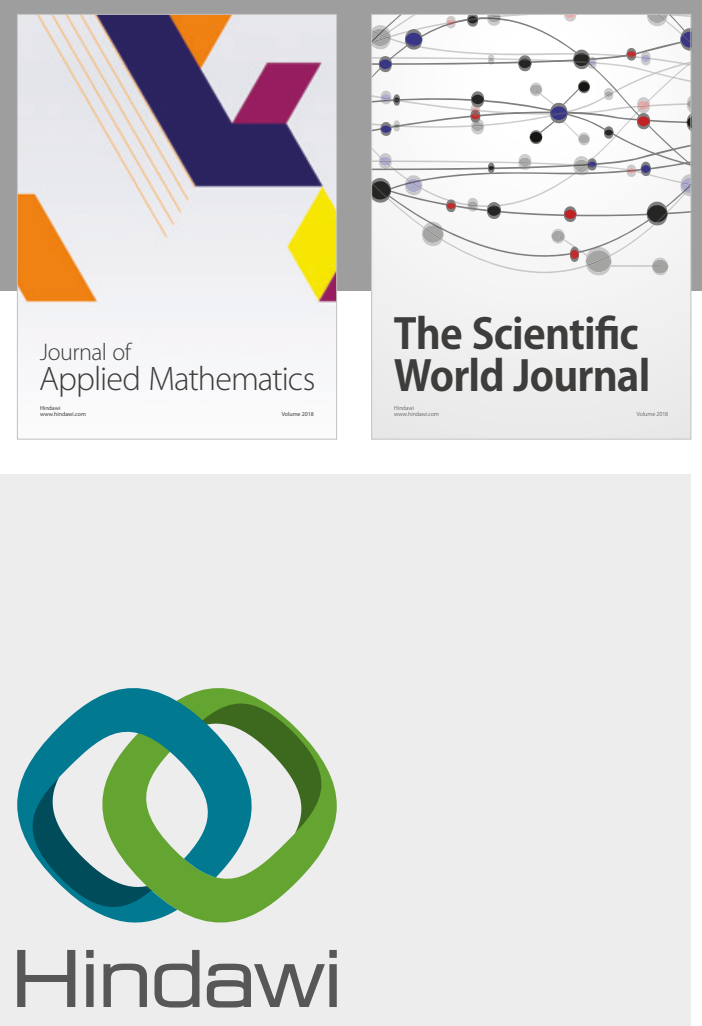

Submit your manuscripts at

www.hindawi.com

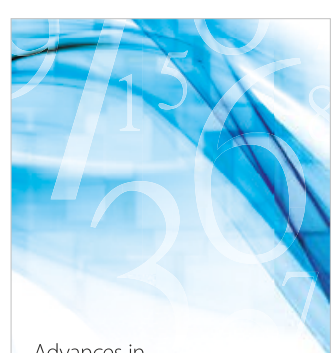

Advances in
Numerical Analysis
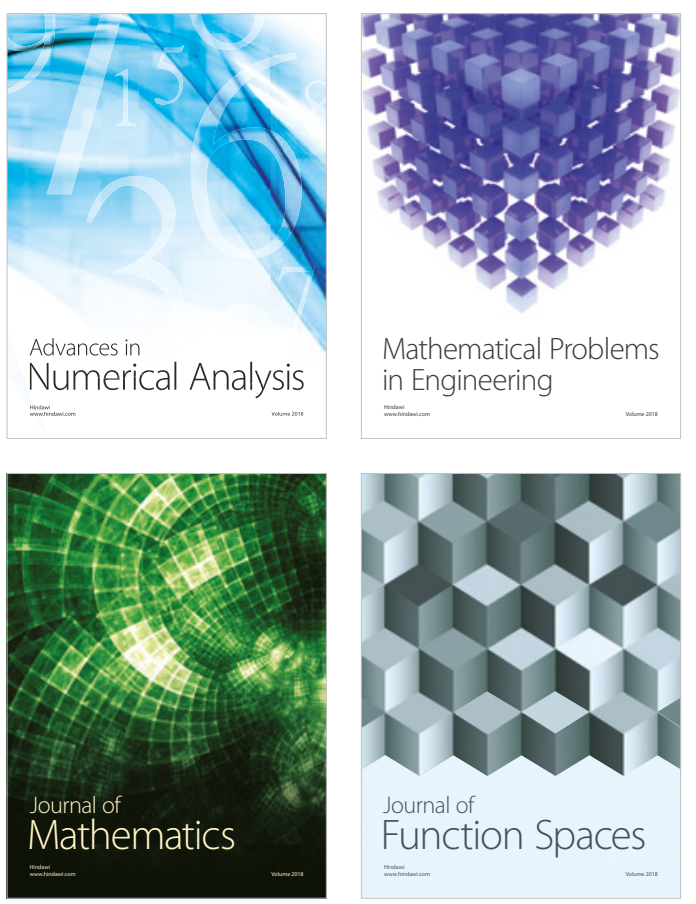

Mathematical Problems in Engineering

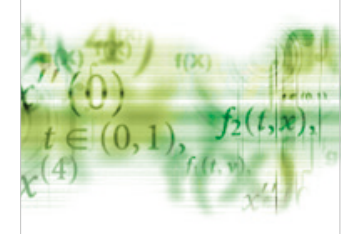

International Journal of

Differential Equations

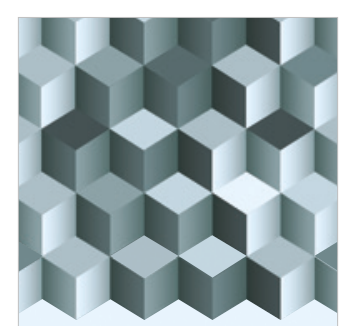

Journal of

Function Spaces
The Scientific

World Journal

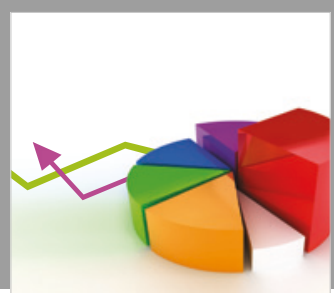

Journal of

Probability and Statistics
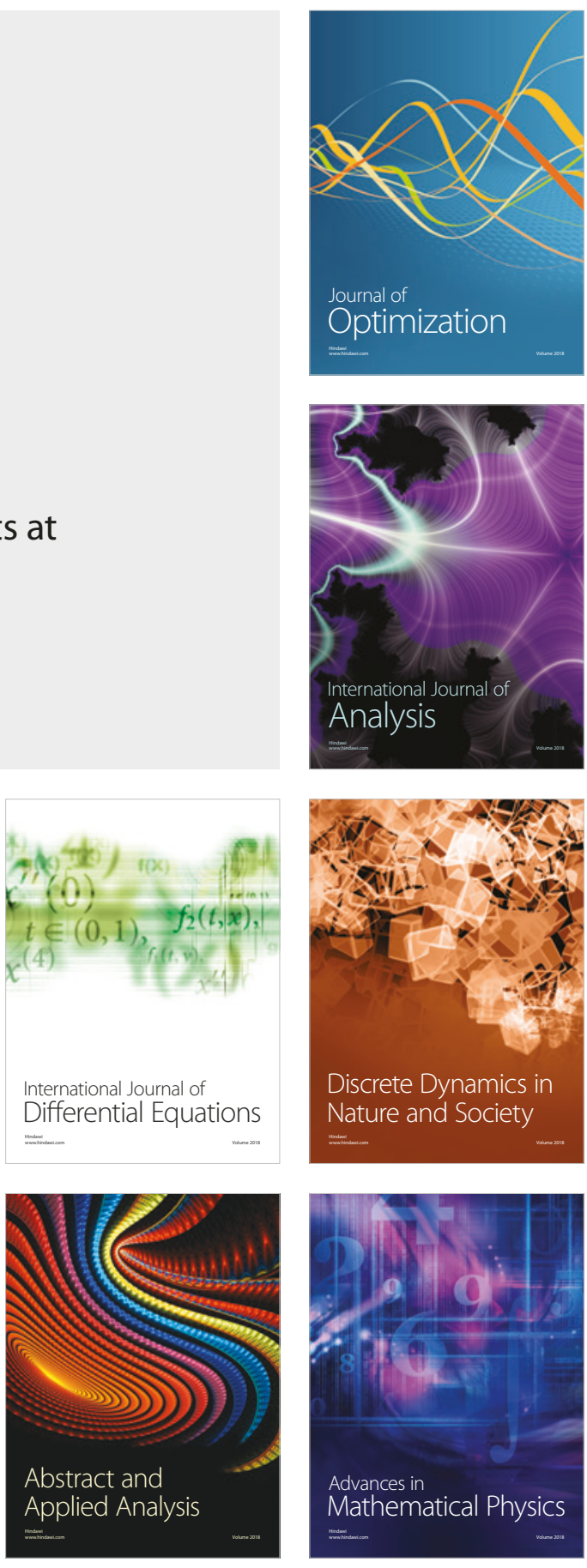